\title{
Radiative properties for warm and hot dense matter
}

\author{
A.G. de la Varga ${ }^{\mathrm{a}, *}$, P. Velarde ${ }^{\mathrm{a}, \mathrm{b}}$, M. Cotelo $^{\mathrm{a}}$, F. de Gaufridy ${ }^{\mathrm{a}, \mathrm{b}}$, P. Zeitoun ${ }^{\mathrm{b}}$ \\ anstituto de Fusión Nuclear, José Gutiérrez Abascal 2, 28006 Madrid, Spain \\ ${ }^{\mathrm{b}}$ Laboratoire d'Optique Appliquée, UMR CNRS/ENSTA ParisTech/Ecole Polytechnique ParisTech, Chemin de la hunière, 91671 Palaiseau, France
}

Keywords:

LTE

Opacities

Radiation transport

\begin{abstract}
A B S T R A C T
We will present calculations of opacities for matter under LTE conditions. Opacities are needed in radiation transport codes to study processes like Inertial Confinement Fusion and plasma amplifiers in Xray secondary sources. For the calculations we use the code BiGBART, with either a hydrogenic approximation with $\mathrm{j}$-splitting or self-consistent data generated with the atomic physics code FAC.

We calculate the atomic structure, oscillator strengths, radiative transition energies, including UTA computations, and photoionization cross-sections. A DCA model determines the configurations considered in the computation of the opacities. The opacities obtained with these two models are compared with experimental measurements.
\end{abstract}

\section{Introduction}

Radiative properties of plasmas are important so that radiation hydrodynamic simulations can produce a physical picture that is as close to reality as possible for both laboratory and astrophysical applications. The simulations are needed to better understand the evolution of these plasmas produced in inertial confinement fusion (ICF) [1], X-ray secondary sources [2] and other laboratory experiments. This field has experienced a major development in the last two decades, allowing a continuous improvement in the accuracy of generated data tables and a better fitting with experimental benchmarks in certain temperature-density ranges.

Even with this development there is still much work to do with respect to atomic structure calculations and line shape modeling for both absorption and emission. Since the 80 s, there is much literature regarding the Screened Hydrogenic Model. These models [3-7] allow very fast computation of total energy, one-electron energies or ionization potentials, suitable for in-line data generation in simulations; however, these models prove too crude when weak lines or spectroscopic accuracy is needed in the spectral analysis. Further, they lack of accuracy for near-neutral atoms, due to the nature of the fitting procedure employed for the screening coefficients.

If we turn to more complex codes [8,9], one finds that for high- $Z$ elements the number of detailed levels required can become computationally prohibitive, especially when several open subshells are present, thus the detailed term accounting treatment is not realistic. Even if we opt for a configuration-average or statistical treatment [10] the number of transitions involved to obtain the atomic data for a single temperature-density point remains large. An alternative is to generate data tables with an acceptable number of grid points in a reasonable time, which transfers the problem to finding the correct balance between accuracy and computational time. Thus, much care is needed in the selection of configurations included in the model.

This holds true for local thermodynamic equilibrium (LTE), if we take a step forward and include out of equilibrium effects (NLTE) within the framework of a collisional-radiative model, the complexity increases yet further. Collisional and radiative rates between connected configurations are needed for solving the system of equations that yields the population of each configuration. Here we focus on LTE conditions and will present recent developments in the development of the opacity code BiGBART used to generate data tables employed in the AMR radiation hydrodynamic simulation code ARWEN [11].

In the following all units are in c.g.s. system and temperatures are in $\mathrm{eV}$ except where noted.

\section{Description of the model}

The atomic physics code BiGBART can be divided in two main parts: atomic structure and frequency dependent opacity calculation. Most of the atomic data can be generated using hydrogenic approximation or using the Flexible Atomic Code [9] package.

\subsection{Atomic structure}

BiGBART is based on the average atom model, widely used to obtain mean properties in hot plasmas. In the frame of the LTE 
theory the state of matter depends only on the temperature and density. If pressure ionization is accounted for with the formula originally proposed by Zimmerman and More [12], the occupation number of the $n_{\kappa}$ orbital is

$P_{n \kappa}=\frac{(2 j+1) D_{n \kappa}}{1+\exp \left(\frac{\tilde{E}_{n \kappa}}{T}+\tilde{\alpha}\right)}$

where $n \kappa$ is the energy level with principal number $n$ and $\kappa=(l-j)(2 j+1), T$ is the plasma temperature, $\tilde{\alpha}=-\mu / T$ is the degeneracy parameter, $\mu$ is the electronic chemical potential, and $D_{n K}$ is a new scaling factor that is a function of a reference density $\rho_{\mathrm{r}}$ depending on the element and the original scaling factor by Zimmerman and More, $d_{n k}$,

$d_{n \kappa}=\frac{1}{1+\left(\alpha \frac{R_{n \kappa}^{0}}{R_{w s}}\right)^{\beta}}$

$D_{n \kappa}=p R+(1-p R) d_{n \kappa}$

$R=\frac{1}{1+\left(\frac{\rho}{\rho_{\mathrm{r}}}\right)^{\gamma}}$

here $R_{n \kappa}{ }^{0}$, or $\langle r\rangle_{n \kappa}$, is the mean radius of orbital $n \kappa$ for the neutral ion, $R_{\text {ws }}$ is the Wigner-Seitz radius, $p$ and $\gamma$ are fitted constants with $p \in$ $[0,1], \alpha$ and $\beta$ are constants fitted so that the average ionization is in good agreement with results from the Thomas-Fermi model.

Isolated ion orbital energies can be obtained with either a relativistic screened hydrogenic model or FAC. New fitted relativistic screening coefficients up to $5 p$ from Ref. [6] were implemented in the calculation of screened charges. The database used to fit this set of universal coefficients was built with energies from the NIST database of experimental atomic energy levels Ref. [13] and energies calculated with FAC. All remaining coefficients were approximated with l-splitting coefficients from Ref. [14].

With the screening coefficients $\sigma_{n \kappa}, n^{\prime} \kappa^{\prime}$, the nuclear charge $Z$, and the level occupation numbers $P_{n k}$, the hydrogenic energies are given by well known equations for the relativistic screened hydrogenic model [15]

$Q_{n \kappa}=Z-\sum_{n^{\prime} K^{\prime}} \sigma_{n \kappa, n^{\prime} \kappa^{\prime}}\left(P_{n^{\prime} \kappa^{\prime}}-\delta_{n \kappa, n^{\prime} \kappa^{\prime}}\right)$

$\varepsilon_{n \kappa}^{0}=\frac{1}{\alpha^{2}}\left\{\left[1+\frac{\left(\alpha Q_{n \kappa}\right)^{2}}{\left(n-\left(j+\frac{1}{2}\right)+\sqrt{\left(j+\frac{1}{2}\right)^{2}-\left(\alpha Q_{n \kappa}\right)^{2}}\right)^{2}}\right]^{-\frac{1}{2}}-1\right\}$

$E_{T}=\sum_{n \kappa} P_{n \kappa} \varepsilon_{n \kappa}^{0}$

$E_{n \kappa}=\left.\frac{\partial E_{T}}{\partial P_{n \kappa}}\right|_{P_{n^{\prime} \kappa^{\prime}}}$

where $Q_{n \kappa}$ and $P_{n \kappa}$ are the effective charge and occupation number of level $n \kappa$, and $\alpha$ is the fine structure constant.
Table 1

Energy levels $\vec{E}_{n K}$ in $\mathrm{eV}$ and average occupation numbers $N_{n x}$ according to a relativistic Hartree-Fock-Slater model compared to BiGBART Hydrogenic and FAC calculations for an iron plasma at $100 \mathrm{eV}$ and $0.1 \mathrm{~g} / \mathrm{cc}$, with $K=(l-j)(2 j+1)$.

\begin{tabular}{|c|c|c|c|c|c|c|c|}
\hline \multirow[t]{2}{*}{$n$} & \multirow[t]{2}{*}{$\kappa$} & \multicolumn{3}{|l|}{$\left|\tilde{E}_{n k}\right|$} & \multicolumn{3}{|l|}{$N_{n \kappa}$} \\
\hline & & HFS & SHM & FAC & HFS & SHM & FAC \\
\hline 1 & -1 & 7534 & 7407 & 7495 & 2.000 & 2.000 & 2.000 \\
\hline 2 & -1 & 1247 & 1229 & 1211 & 1.997 & 1.996 & 1.995 \\
\hline 2 & 1 & 1144 & 1109 & 1103 & 1.991 & 1.988 & 1.986 \\
\hline 2 & -2 & 1132 & 1099 & 1090 & 3.979 & 3.974 & 3.970 \\
\hline 3 & -1 & 395.9 & 411.2 & 403.1 & 0.215 & 0.270 & 0.241 \\
\hline 3 & 1 & 363.1 & 376.4 & 370.3 & 0.160 & 0.199 & 0.180 \\
\hline 3 & -2 & 361.0 & 375.1 & 367.5 & 0.313 & 0.393 & 0.351 \\
\hline 3 & 2 & 313.6 & 329.0 & 319.0 & 0.201 & 0.257 & 0.224 \\
\hline 3 & -3 & 313.1 & 328.7 & 318.5 & 0.300 & 0.385 & 0.334 \\
\hline 4 & -1 & 171.7 & 186.6 & 176.7 & 0.025 & 0.030 & 0.028 \\
\hline 4 & 1 & 158.8 & 171.7 & 163.8 & 0.022 & 0.025 & 0.024 \\
\hline 4 & -2 & 158.2 & 171.3 & 162.7 & 0.044 & 0.050 & 0.049 \\
\hline 4 & 2 & 140.5 & 150.3 & 144.5 & 0.037 & 0.018 & 0.041 \\
\hline 4 & -3 & 140.4 & 150.2 & 144.4 & 0.056 & 0.027 & 0.061 \\
\hline 4 & 3 & 127.0 & 146.3 & 135.9 & 0.049 & 0.001 & 0.056 \\
\hline 4 & -4 & 126.9 & 146.2 & 135.8 & 0.065 & 0.002 & 0.075 \\
\hline 5 & -1 & 82.37 & 80.98 & 83.51 & 0.010 & 0.0014 & 0.011 \\
\hline 5 & 1 & 76.24 & 80.11 & 77.22 & 0.010 & 0.0005 & 0.010 \\
\hline 5 & -2 & 76.00 & 79.96 & 76.72 & 0.020 & 0.0011 & 0.020 \\
\hline 5 & 2 & 67.57 & 78.47 & 67.99 & 0.018 & 0.0002 & 0.019 \\
\hline 5 & -3 & 67.51 & 78.42 & 67.89 & 0.027 & 0.0003 & 0.028 \\
\hline 5 & 3 & 61.17 & 77.43 & 63.55 & 0.025 & 0.0001 & 0.027 \\
\hline 5 & -4 & 61.14 & 77.41 & 63.52 & 0.034 & 0.0002 & 0.036 \\
\hline 5 & 4 & 58.29 & 78.17 & 61.55 & 0.033 & 0.0002 & 0.036 \\
\hline 5 & -5 & 58.28 & 78.16 & 61.53 & 0.041 & 0.0003 & 0.045 \\
\hline
\end{tabular}

The FAC/Hydrogenic orbital energies are corrected with an interpolated ionization potential lowering model based on the work by Ref. [16] fit by More [17], in eV

$\Delta E=2.16 \times 10^{-7} \frac{\bar{Z}}{R_{\mathrm{ws}}}\left[\left(1+\left(\frac{\lambda_{\mathrm{D}}}{R_{\mathrm{ws}}}\right)^{3}\right)^{2 / 3}-\left(\frac{\lambda_{D}}{R_{\mathrm{ws}}}\right)^{2}\right]$

where $\lambda_{\mathrm{D}}$ is the Debye length and $R_{\mathrm{ws}}$ the Wigner-Seitz radius. This expression interpolates between strongly and weakly coupled plasma conditions. The drawback of this type of continuum lowering corrections is that the abrupt truncation in the energy levels leads to thermodynamic inconsistencies.

In Table 1 we show a comparison of average atom computations with a Hartree-Fock-Slater model [15] and BiGBART. FAC selfconsistent computations are in better agreement, especially for outer shells ( $n \geq 5$ though only $n=5$ is shown in the table) where hydrogenic screened charges obtained increase with $l$ thus leading to higher binding energies inverting their expected values.

To improve the convergence of the average atom iteration at low temperatures, typically below $5 \mathrm{eV}$, we included the iteration algorithm by Mancini and Fontán [18]. Considering all levels up to principal quantum number $n=10$, we need to calculate $100 \mathrm{occu}$ pation numbers provided that the energy levels $E_{n \kappa}$ and the degeneracy parameter are known. Under the assumption of uniform free electron density, the average ionization $\bar{Z}$ can be computed with the formula

$f_{1 / 2}(\tilde{\alpha})=2.79 \times 10^{-3} \frac{\rho \bar{Z}}{A T 32}$

where $f_{1 / 2}$ is the Fermi-Dirac integral of order $1 / 2, \rho$ is the density in $\mathrm{g} / \mathrm{cm}^{3}, T$ is the temperature in $\mathrm{keV}$, and $A$ is the atomic mass of the element. For a given element and fixed density and temperature, the average ionization $\bar{Z}$ depends only on the degeneracy parameter $\tilde{\alpha}=\mu / T$. The energy levels and degeneracy parameter also depend on the occupation numbers. Therefore, to compute 
these energy levels, degeneracy parameter and occupation numbers self-consistently we need to solve a set of 101 non-linear equations. During the iteration, instead of setting the occupation number to 0 whenever $E_{n k}>0$, the state of bound and unbound levels between consecutive iterations is stored. This way the information is not lost when the outermost levels tend to jump in and out of the continuum due to oscillations in the degeneracy parameter in the first few iterations for low temperatures.

When the convergence criterion is reached in all levels, we check if this solution satisfies the charge neutrality condition

$n(\tilde{\alpha})=\sum_{n \kappa} P_{n \kappa}\left(\tilde{E}_{n \kappa}, \tilde{\alpha}\right)+\bar{Z}(\tilde{\alpha})-Z=0$

Knowing the dependence of the occupation numbers and ionization on $\tilde{\alpha}$, a Newton-Raphson method can be applied to increase convergence between iterations in this external loop, so the initial $\tilde{\alpha}$ in the next iteration will be

$\tilde{\alpha}^{(k+1)}=\tilde{\alpha}^{(k)}-\left.\frac{n(\tilde{\alpha})}{n^{\prime}(\tilde{\alpha})}\right|_{\tilde{\alpha}^{(k)}}$

\subsection{Frequency dependent opacity}

For the absorption coefficient we consider contributions from photoabsorption, photoionization, inverse Bremsstrahlung and Thomson scattering processes.

\subsubsection{Photoabsorption (bound-bound)}

The contribution to the mass absorption coefficient coming from bound-bound transitions between levels $i=n \kappa$ and $f=n^{\prime} \kappa^{\prime}$ for a specific photon energy $h v$ is

$\kappa^{b b}=\frac{2 \pi^{2} \alpha a_{0}^{2} N_{A}}{A} \sum_{i, f} f_{i, f} \Psi_{i, f}$

where $\alpha$ is the fine structure constant, $a_{0}$ is the Bohr radius, $\Psi_{i f}$ is the line shape function that includes all the broadening effects considered, and $f_{i, f}$ is the oscillator strength of the transition. In the hydrogenic approximation

$f_{i, f}^{\mathrm{H}}=\frac{1}{3} \Delta E_{i, f}\left(2 j^{\prime}+1\right)\left(l+l^{\prime}+1\right) W^{2}\left(l^{\prime} j^{\prime} l j ; \frac{1}{2} 1\right) r_{n l, n^{\prime} t^{\prime}}^{2}$

here $W\left(l^{\prime} j^{\prime} l j ; 1 / 21\right)$ is the Racah coefficient and $r_{n l, n^{\prime} l^{\prime}}$ is the Dipole matrix element

$r_{n l, n^{\prime} l^{\prime}}=\int_{0}^{r_{0}} R_{n l}(r) r R_{n^{\prime} l^{\prime}}(r) \mathrm{d} r$

When we use FAC, we obtain the weighted oscillator strength of the radiative transition and, with the $2 J_{i}$ or the statistical weight of the initial state

$f_{i, f}=P_{i}\left(1-\frac{P_{f}}{2 j^{\prime}+1}\right) f_{i, f}^{\mathrm{H}}$

$f_{i, f}=\frac{g f}{2 J_{i}+1}$

For the line shape function $\Psi_{i, f}$ we use a Voigt profile that incorporates both Gaussian and Lorentzian broadening effects. In Eq. (15) $\Delta_{i, f}$ is the Doppler and UTA Gaussian contribution, and $\gamma_{i, f}$ is the homogeneous width comprising the collisional and natural broadenings.

$\Psi_{i, f}=\frac{1}{\sqrt{\pi} \Delta_{i, f}} K\left(\frac{h \nu-\Delta E_{i, f}}{\Delta_{i, f}}, \frac{\gamma_{i, f}}{\Delta_{i, f}}\right)$
$\Delta_{i f f}=\sqrt{\sigma_{\mathrm{UTA}}^{2}+\sigma_{\mathrm{D}}^{2}}$

$\gamma_{i, f}=\gamma^{\mathrm{N}}+\gamma^{\mathrm{C}}$

For the Doppler $\sigma_{\mathrm{D}}$ and natural $\gamma^{\mathrm{N}}$ widths we use standard formulas that can be found in Ref. [19]. For the collisional broadening $\gamma^{\mathrm{C}}$ only the upper level is considered, with a formula by Armstrong et al. [20]. We use screened nuclear charges instead of effective principal quantum numbers.

$\sigma_{\mathrm{D}}=2.44 \times 10^{-3} \Delta E_{i, f}\left(\frac{T}{A}\right)^{1 / 2}$

$\gamma^{N}=4.75 \times 10^{-6}\left(\Delta E_{i, f}\right)^{2}$

$\gamma^{\mathrm{C}}=1.268 \times 10^{-3} \frac{\bar{Z} \rho}{A T^{1 / 2}}\left\langle r_{\mathrm{f}}^{2}\right\rangle$

where $\left\langle r_{\mathrm{f}}^{2}\right\rangle$ is the mean square radius of the final state and is calculated with recurrence formulas by Andrae [21].

\subsubsection{Photoionization (bound-free)}

In the hydrogenic approximation the cross-section for the photoionization process and hence the mass absorption coefficient, can be derived from the formula for $\kappa_{\mathrm{bb}}$ by replacing the bound wave functions for the final state $n^{\prime} \kappa^{\prime}$ by a continuum wave function with kinetic energy $\varepsilon^{\prime}$ denoted as $c=\kappa^{\prime}$. The sum over the quantum numbers $n^{\prime}$ must be replaced by the integral over the energy $\varepsilon^{\prime}$. This yields the final expression

$\kappa^{\mathrm{bf}}=\frac{2 \pi^{2} \alpha a_{0}^{2} N_{\mathrm{A}}}{A} \sum_{i, c} P_{i} \int_{\varepsilon_{0}}^{\infty}\left(1-n_{\varepsilon^{\prime}}\right) \delta\left(h \nu-\varepsilon^{\prime}+\tilde{E}_{i}\right) f_{i, c} \mathrm{~d} \varepsilon^{\prime}$

with

$f_{i, c}=\frac{1}{3}\left(\varepsilon^{\prime}-\tilde{E}_{i}\right)\left(2 j^{\prime}+1\right)\left(l+l^{\prime}+1\right) W^{2}\left(l^{\prime} j^{\prime} l j ; \frac{1}{2} 1\right) r_{n l, \varepsilon^{\prime} l^{\prime}}^{2}$

$r_{n l, \varepsilon^{\prime} t^{\prime}}=\int_{0}^{r_{0}} R_{n l}(r) r R_{\varepsilon^{\prime} t^{\prime}}(r) \mathrm{d} r$

$n_{\varepsilon}$ is the distribution function of free electrons

$n_{\varepsilon}=\frac{1}{1+\exp \left(\frac{\varepsilon-\mu}{T}\right)}$

When using FAC, the photoionization cross-section is obtained from a fitting formula and tabulated values of the weighted boundfree differential oscillator strength.

\subsubsection{Inverse Bremsstrahlung (free-free)}

For inverse Bremsstrahlung we use the result of the hydrogen atom in the same way as in Ref. [19] and replace the nuclear charge by the ionization state

$\kappa^{\mathrm{ff}}=2.78\left(\frac{\bar{Z}^{3} \rho}{A^{2} T^{1 / 2}} \frac{1}{(h \nu)^{3}}\right) g^{\mathrm{ff}} \Phi$

The Gaunt factor $g^{\text {ff }}$ contains quantum-mechanical corrections to the classical result and was calculated by Menzel and Pekeris [22] 
$g^{\mathrm{ff}}=1+0.1728\left(\frac{h \nu}{13.6 \times 10^{-3} Z^{2}}\right)^{1 / 3}\left(1+2 \frac{T}{h v}\right)$

When the density is very high, the distribution is no longer a Maxwellian and the absorption coefficient must be averaged over a Fermi-Dirac distribution to take into account the degeneration effects. Therefore, the absorption coefficient is multiplied by a factor from Cox and Giuli [23]

$\Phi=\frac{\frac{\pi^{1 / 2}}{2} \ln \left(\frac{1+\exp (-\tilde{\alpha})}{1+\exp \left(-\tilde{\alpha}-\frac{h \nu}{T}\right)}\right)}{f_{1 / 2}(\tilde{\alpha})\left[1-\exp \left(-\frac{h \nu}{T}\right)\right]}$

\subsubsection{Scattering}

For the scattering coefficient, the Thomson cross-section is used and only scattering due to free electrons is considered

$\kappa^{\mathrm{es}}=0.4 \frac{\bar{Z}}{A}$

Now, with all the formulas necessary to calculate the frequency dependent opacity, we must take into account the induced emission to obtain the total absorption coefficient [24]. If LTE conditions prevail this leads to the final equation for the absorption coefficient:

$\kappa=\left[\kappa^{\mathrm{bb}}+\kappa^{\mathrm{bf}}+\kappa^{\mathrm{fff}}\right]\left[1-\exp \left(-\frac{h \nu}{T}\right)\right]+\kappa^{\mathrm{es}}$

\subsection{Detailed configuration accounting model}

A detailed configuration is defined by a set of integer values for each orbital $n \kappa$. The configurations are generated using the average atom occupation numbers as a statistical mean. We start by setting the non-integer average atom occupation numbers to their closest integer value to obtain the most probable ion. Then we set a maximum and minimum integer occupation value for each orbital, this is established by using a preset parameter that represents a preset deviation from the average value. Those orbitals that are fully populated or are very close to the orbital's degeneracy are considered closed, with a fixed value of $2 j+1$.

All the possible combinations in orbital occupation numbers are gathered depending on the ionization state $k$ and included in a Saha-approximated system as described in Ref. [15], with the most probable ion energy $E_{\mathrm{mp}}$ as the reference.

$p_{i k}=\frac{p_{k}}{Q_{k}} g_{i k} \exp \left(-\frac{E_{i k}-E_{\mathrm{mp}}}{T}\right)$

$Q_{k}=\sum_{i \in k} g_{i k} \exp \left(-\frac{E_{i k}-E_{\mathrm{mp}}}{T}\right)$

where $E_{i k}, g_{i k}$ and $p_{i k}$ are the energy, statistical weight and probability of the configuration $i$ in the ionization state $k, p_{k}$ is the fraction of ionization state $k, \Omega_{k}$ is the partition function of ionization state $k$ and $T$ is the temperature.

\section{Comparison with experimental data}

In order to check the accuracy of our model we have compared our theoretical results with experimental measurements, for low-,

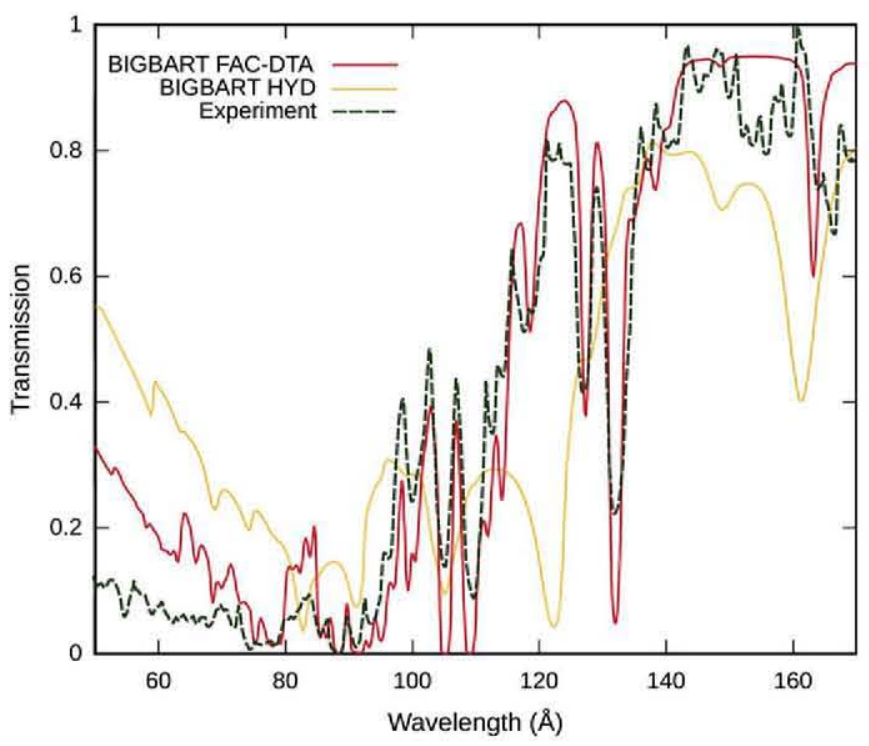

Fig. 1. Experimental transmission of $\mathrm{Al}$ compared with BiGBART transmission spectrum for a temperature of $20 \mathrm{eV}$ and a density of $10 \mathrm{mg} / \mathrm{cc}$ with a convolution equivalent to a $1 \mathrm{eV}$ experimental broadening. Dominant $2 \mathrm{p}-3 \mathrm{~s}$ multiplets of $\mathrm{Al}^{4+}$ and $\mathrm{Al}^{5+}$ can be seen in the 115-135 and 100-112 region, respectively.

medium- and high-Z elements $[25,26]$. For this we need accurate measurements at a well-defined temperature and density.

\section{1. $\mathrm{Al}$}

For low $\mathrm{Z}$ we chose aluminum from the work of Winhart [25]. The experimental conditions for aluminium were determined with hydrodynamics simulations, yielding a sample with a density of $10 \mathrm{mg} / \mathrm{cc}$ and a temperature of $20 \mathrm{eV}$ at the time of measurement. For aluminum we show detailed calculations performed with FAC, which due to the lower $\mathrm{Z}$ has a tractable number of detailed levels for the DTA calculation.

This results, displayed in Fig. 1, show good agreement with line positions of the partially resolved $2 \mathrm{p}-3 \mathrm{~s}$ multiplets of $\mathrm{Al}^{4+}$

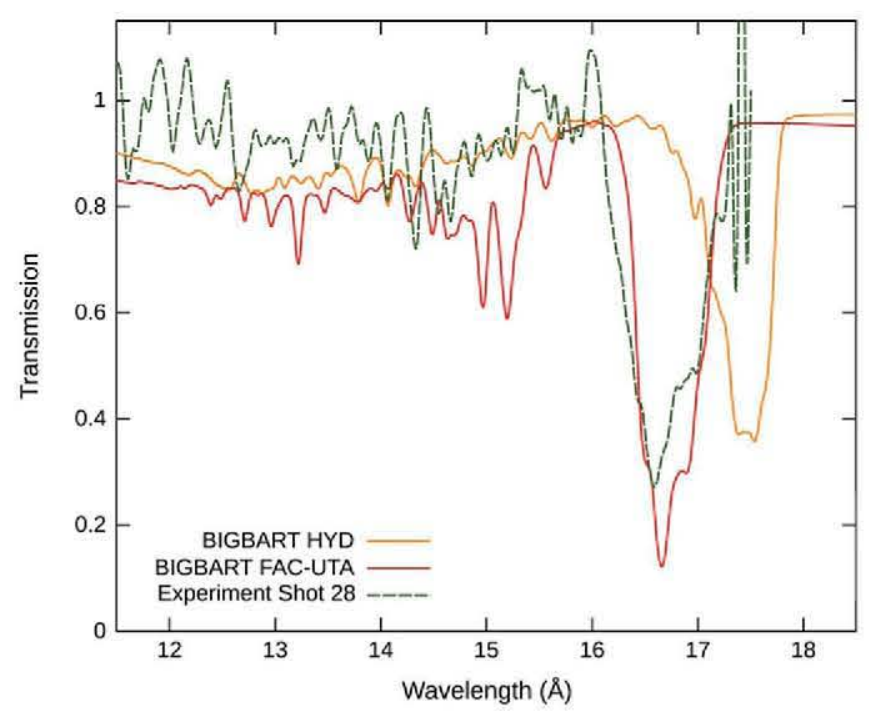

Fig. 2. Experimental transmission of Fe compared with BiGBART transmission spectrum for a temperature of $22 \mathrm{eV}$ and a density of $4 \mathrm{mg} / \mathrm{cc}$ with an experimental broadening of $4 \mathrm{eV}$. 


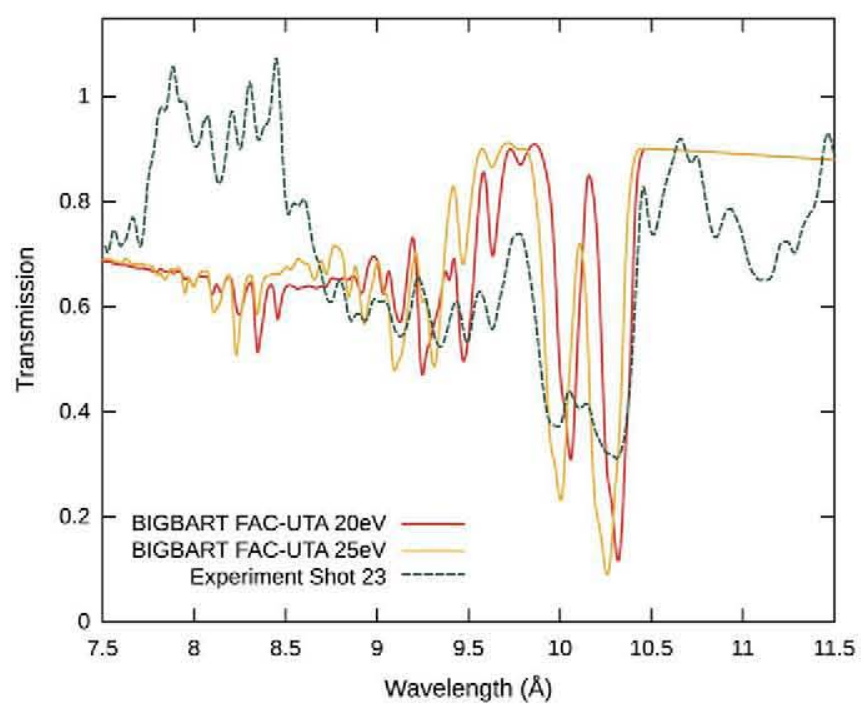

Fig. 3. Experimental transmission of Ge compared with BiGBART FAC transmission spectrum for two temperatures, $20 \mathrm{eV}$ and $25 \mathrm{eV}$, and a density of $10 \mathrm{mg} / \mathrm{cc}$ with an experimental broadening of $4 \mathrm{eV}$.

between 115 and $135 \AA$, and $\mathrm{Al}^{5+}$ between 100 and $112 \AA$, which are the dominant absorption lines as seen in the original article. The approximate formulas used in the natural and collision line broadening might have be the cause of the underestimation of some of the line widths, leading to higher absorption peaks. The hydrogenic approximation shows poor agreement, especially in the $\mathrm{Al}^{4+} 2 \mathrm{p}-3$ s transitions.

\section{2. $\mathrm{Fe}$}

For medium $\mathrm{Z}$ we chose the iron experiment from Loisel [26]. The hydrodynamics simulations determined the approximate conditions yielding a density of $4 \mathrm{mg} / \mathrm{cc}$ and a temperature of $22 \mathrm{eV}$. The computations include a convolution of a Gaussian width of $4 \mathrm{eV}$ to simulate the instrumental broadening.

With a single density-temperature point, BiGBART reasonably well reproduces the L-shell transitions as shown in Fig. 2, especially the main absorption window $2 \mathrm{p}-3 \mathrm{~d}$ transitions, between 16 and

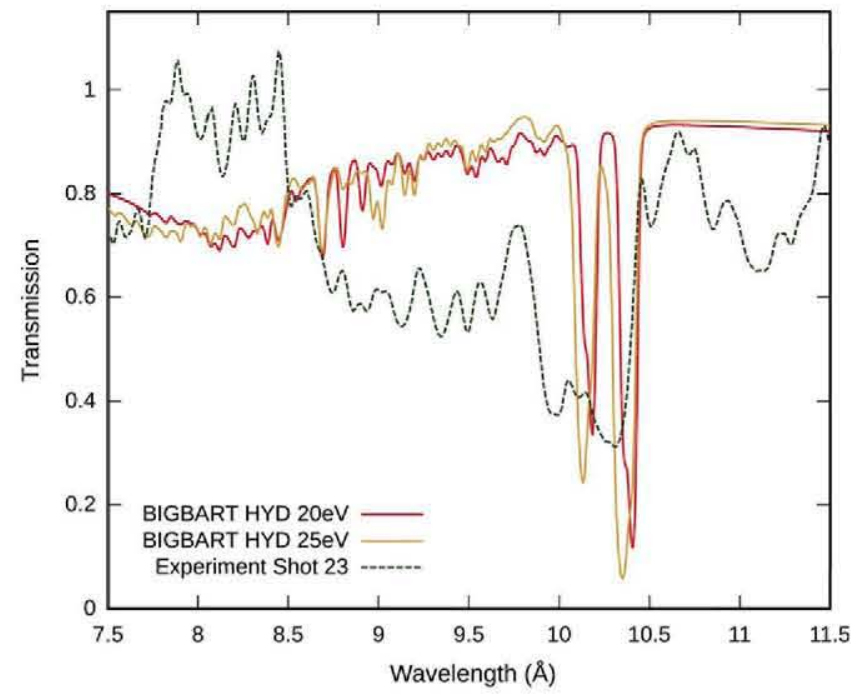

Fig. 4. Experimental transmission of Ge compared with BiGBART Hydrogenic transmission spectrum for two temperatures, $20 \mathrm{eV}$ and $25 \mathrm{eV}$, and a density of $10 \mathrm{mg} / \mathrm{cc}$ with an experimental broadening of $4 \mathrm{eV}$.

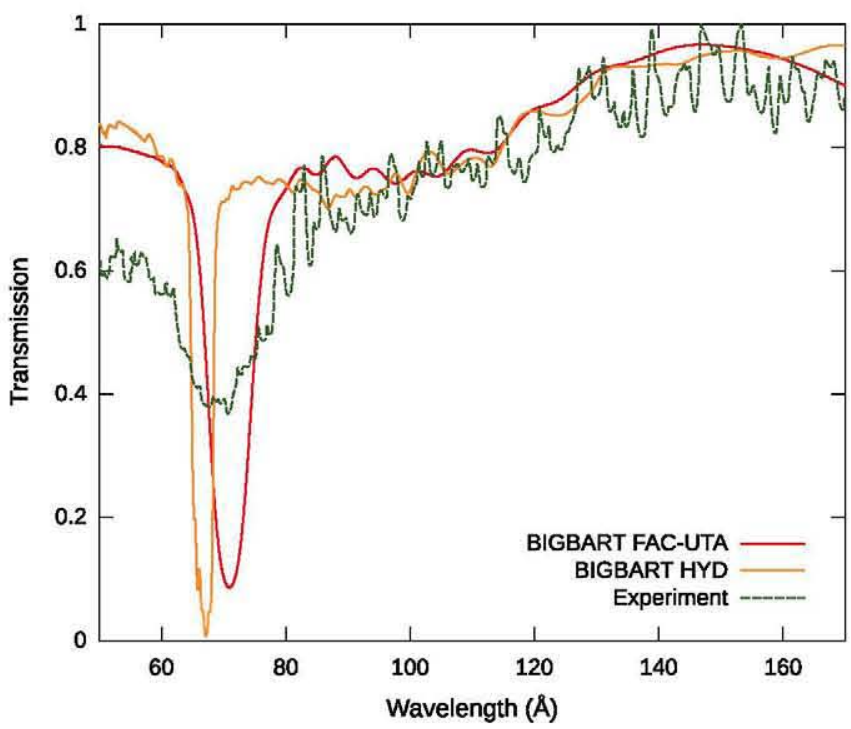

Fig. 5. Experimental transmission of Ho compared with BiGBART transmission spectrum for a temperature of $20 \mathrm{eV}$ and a density of $10 \mathrm{mg} / \mathrm{cc}$ with an experimental broadening of $1 \mathrm{eV}$.

$17.5 \AA$. As stated in the original article, accounting for temperature gradients should improve the width of this absorption band. Again, some of the 2 p transition widths seem underestimated, especially those around $15 \AA$. The hydrogenic approximation is in worse agreement predicting a $2 \mathrm{p}-3 \mathrm{~d}$ window shifted towards lower energies. However, as the hydrogenic wave functions produce acceptable results for dominant transitions dipole matrix elements but fails to reproduce transitions with smaller values [15] seen in the lower wavelength absorption part of the spectrum, from 12 to $16 \AA$.

\section{3. $\mathrm{Ge}$}

For medium $\mathrm{Z}$ we carried out computations for the germanium case also from the article by Loisel [26]. Simulations determined higher gradients in temperature and density than in the iron experiment. The conditions for $\mathrm{Ge}$ calculated here are for a density of $10 \mathrm{mg} / \mathrm{cc}$ and the two temperatures $20 \mathrm{eV}$ and $25 \mathrm{eV}$, with an instrumental resolution of $4 \mathrm{eV}$.

BiGBART FAC computations in Fig. 3 show the two main components of the $2 p-3 d$ split array between 9.75 and $10.5 \AA$ that appear due to a slightly higher $Z$ compared to iron. These results are similar to those found in Loisel [26], where reproducing the shallow dip without accounting for the relatively strong gradients was not possible. On the contrary, the hydrogenic results shown in Fig. 4 display shifted and narrower line positions of the $2 \mathrm{p}-3 \mathrm{~d}$ band and seriously underestimates the $2 \mathrm{p}-4 \mathrm{~d}$ line strengths resulting in very poor overall agreement.

\section{4. $\mathrm{Ho}$}

For high $\mathrm{Z}$ we show computations for holmium and compare them with experimental data extracted from Winhart [25] in which a plasma with a density of $10 \mathrm{mg} / \mathrm{cc}$ and a temperature of $20 \mathrm{eV}$ was presented. Further, the reported instrumental resolution is $1 \mathrm{eV}$.

In this case, the main feature to be observed is the $4 \mathrm{~d}-4 \mathrm{f}$ window at $75 \AA$. Both the FAC and Hydrogenic, as seen in Fig. 5 computations reproduce this window, though the Hydrogenic case shows a slightly shifted and narrower transition band. The long wavelength part of the spectrum, above $100 \AA$, where boundfree absorption prevails is well described by both models. 


\section{Conclusion}

We have checked developments in the atomic code used to generate opacity data tables with experimental measurements. Some of the experiments selected [26] featured temperature and density gradients so a full comparison through a single temperature-density point was not possible. In spite of all this, it can be seen that new models implemented show better agreement with experiments compared to the existing hydrogenic model. Although BiGBART is not aimed for spectroscopic purposes there is still room for improvement, especially in the broadening models included or the extension to NLTE bias a steady state collisional-radiative model. Future work will be in this direction.

\section{Acknowledgements}

One of the authors (A.G.V.) would like to thank Dr. Mendoza for providing the relativistic screening coefficients. The authors would like to thank the financial support provided by the Spanish Ministerio de Educación y Cultura program no. ENE2009-09837 and also to the European Commission projects HiPER-PP (211737) and SFINX-LASERLAB-II.

\section{References}

[1] E.I. Moses, Fusion Eng. Des. 85 (2010) 983-986.

[2] P. Zeitoun, et al., Nature (2004) 426-429.

[3] R.M. More, J. Quant. Spectrosc. Radiat. Transfer 27 (1982).
[4] F. Perrot, Phys. Scr. 39 (1989) 332-337.

[5] G. Faussurier, C. Blancard, A. Decoster, J. Ouant. Spectrosc Radiat. Transfer 58 (1997) 233.

[6] M.A. Mendoza, J.G. Rubiano, J.M. Gil, R. Rodríguez, R. Florido, P. Martel, E. Mínguez, 36th EPS Conference on Plasma Physics Sofia (2009).

[7] C.C. Smith, A sreened hydrogenic model with fine structure splitting, High Energy Density Phys. (2010).

[8] A. Bar-Shalom, M. Klapisch, J. Oreg, J. Quant. Spectrosc. Radiat. Transfer 71 (2001) 169-188.

[9] M. Gu, Astrophys. J. 582 (2003) 1241.

[10] C. Bauche-Arnault, J. Bauche, M. Klapisch, Phys. Rev. A 20 (1979) 2424-2439.

[11] F. Ogando, P. Velarde, J. Quant. Spectrosc. Radiat. Transfer 71 (2001) 541-550.

[12] G.B. Zimmerman, R.M. More, J. Quant. Spectrosc. Radiat. Transfer 23 (1979) $517-522$.

[13] Y. Ralchenko, A.E. Kramida, J. Reader, N.A. Team, NIST Atomic Spectra (2008)

[14] G. Faussurier, C. Blancard, P. Renaudin, High Energy Density Phys. 4 (2008) 114-123.

[15] A.F. Nikiforov, V.G. Novikov, V.B. Uvarov, Quantum-Statistical Models of Hot Dense Matter. Birkh Ãuser, 2005.

[16] J.C. Stewart, K.D. Pyatt Jr., Astrophys. J. 144 (1966) 1203.

[17] R.M. More, Applied Atomic Collision Physics, vol. 2, Academis Press, New York, 1982.

[18] R.C. Mancini, C.F. Fontán, J. Quant. Spectrosc. Radiat. Transfer 34 (1985) 115-122.

[19] A. Rickert, J. Meyer-Ter-Vehn, Laser Part. Beams 8 (1990) 715-727.

[20] B.H. Armstrong, et al., Opacity of High Temperature Air, in Progress in High Temperature Physics and Chemistry, vol. 1, Pergamon, Oxford, 1966.

[21] A. Andrae, J. Phys. B: At. Mol. Opt. Phys. 30 (1997) 4435-4451.

[22] D.H. Menzel, C.H. Pekeris, Mon. Not. R. Astron. Soc. 96 (1935).

[23] P.J. Cox, R.T. Giuli, Principles of Stellar Structure, vol. 1, Gordon and Breach, New York, 1968.

[24] Y.B. Zel'dovich, Y.P. Raizer, Physics of Shock Waves and High-temperature Hydrodynamic Phenomena. Academic Press, San Diego, 1966.

[25] G. Winhart, et al., J. Quant. Spectrosc. Radiat. Transfer 54 (1995) 437-446

[26] G. Loisel, et al., High Energy Density Phys. 5 (2009) 173-181. 